\title{
Generation of tunable visible picosecond pulses by frequency-doubling of a quantum-dot laser in a PPKTP waveguide
}

\author{
Ksenia A. Fedorova*a ${ }^{\mathrm{a}}$, Grigorii S. Sokolovskii ${ }^{\mathrm{a}, \mathrm{b}}$, Daniil I. Nikitichev ${ }^{\mathrm{a}}$, Philip R. Battle ${ }^{\mathrm{c}}$, \\ Daniil A. Livshits ${ }^{\mathrm{d}}$, Edik U. Rafailov ${ }^{\mathrm{a}}$ \\ ${ }^{a}$ Photonics \& Nanoscience Group, University of Dundee, Nethergate, Dundee, DD1 4HN, UK; \\ ${ }^{\mathrm{b}}$ Ioffe Physico-Technical Institute, 26 Polytechnicheskaya str., St. Petersburg, 194021, Russia; \\ ${ }^{c}$ AdvR Inc., 2311 S. 7th Avenue, Building No. 1, Bozeman, Montana, 59715, USA; \\ ${ }^{\mathrm{d}}$ Innolume GmbH, 11 Konrad-Adenauer-Allee, Dortmund, 44263, Germany
}

\begin{abstract}
We demonstrate a compact all-room-temperature picosecond laser source broadly tunable in the visible spectral region between $600 \mathrm{~nm}$ and $627 \mathrm{~nm}$. The tunable radiation is obtained by frequency-doubling of a tunable quantum-dot external-cavity mode-locked laser in a periodically-poled KTP multimode waveguide. In this case, utilization of a significant difference in the effective refractive indices of the high- and low-order modes enables to match the period of poling in a very broad wavelength range. The maximum achieved second harmonic output peak power is $3.25 \mathrm{~mW}$ at $613 \mathrm{~nm}$ for $71.43 \mathrm{~mW}$ of launched pump peak power at $1226 \mathrm{~nm}$, resulting in conversion efficiency of $4.55 \%$.
\end{abstract}

Keywords: Tunable lasers, Visible lasers, Second harmonic generation

\section{INTRODUCTION}

Widely tunable compact laser sources emitting light with picosecond pulse duration in the visible spectral region are of considerable interest for various applications, such as biophotonics [1], photomedicine [2,3] and laser-projection displays [4]. An attractive method for the realization of portable visible laser sources is frequency-doubling of infrared light in a nonlinear crystal containing a waveguide [5]. In this respect, the recent availability of low-cost, good quality InAs/GaAs quantum-dot (QD) lasers, allowing the coverage of a broad spectral range between $1.1 \mu \mathrm{m}$ and $1.3 \mu \mathrm{m}[6]$, in combination with well-established techniques to fabricate good quality waveguides in nonlinear crystals, enables compact tunable CW laser sources in the visible spectral region to be realized [7]. Furthermore, QD materials have shown a great promise for ultrafast technology [8], thus making quantum-dot external-cavity mode-locked diode lasers (QD-ECMLLs) [9] excellent candidates for the development of compact room-temperature tunable ultrashort pulse sources in the visible spectral region based on second harmonic generation (SHG). Such sources can be based on a single laser diode and a single crystal waveguide and can be realized by utilization of a significant difference in the effective refractive indices of the high-order and low-order modes in multimode waveguides. This concept enables one to shift the difference between the effective refractive indices of the fundamental and SHG waves to match the period of poling in a very broad wavelength range.

In this work we demonstrate a compact all-room-temperature laser source broadly tunable in the visible spectral region $(600 \mathrm{~nm}-627 \mathrm{~nm})$ in the picosecond regime. The tunable radiation is obtained by SHG in a periodically-poled potassium titanyl phosphate (PPKTP) waveguide using a tunable QD-ECMLL. The maximum SHG output peak power of $3.25 \mathrm{~mW}$ at $613 \mathrm{~nm}$ is achieved for $71.43 \mathrm{~mW}$ of launched pump peak power at $1226 \mathrm{~nm}$, resulting in conversion efficiency of $4.55 \%$. The demonstrated concept opens-up a new avenue for an order-of-magnitude increase in the wavelength tuning range for frequency conversion from a single crystal.

*K.A.Fedorova@dundee.ac.uk; phone +44 (01382) 386571; fax +44 (01382) 384389

Nonlinear Frequency Generation and Conversion: Materials, Devices, and Applications XII edited by Konstantin L. Vodopyanov, Proc. of SPIE Vol. 8604, 860406 (C) 2013 SPIE · CCC code: $0277-786 X / 13 / \$ 18 \cdot$ doi: $10.1117 / 12.2002869$ 


\section{TUNABLE SECOND HARMONIC GENERATION}

The idea of enabling broadly tunable SHG used in this work is based on the utilization of a significant difference in the effective refractive indices of the high-order and low-order modes in the waveguide [7], that enables one to shift the difference between the effective refractive indices of the fundamental and second harmonic waves to match the poling period in very broad wavelength range limited mainly by the waveguide refractive index step, as shown schematically in Figure 1 (a).
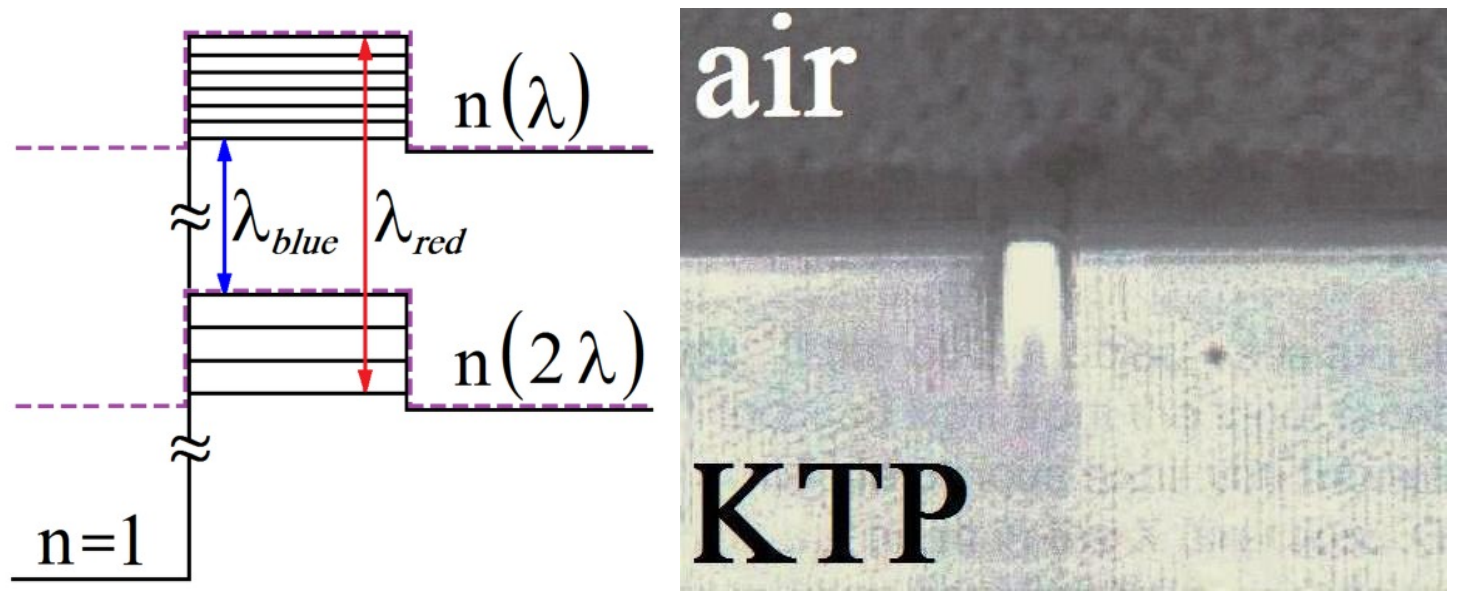

Figure 1. (a) Simplified schematic of the effective refractive indices for the fundamental and second harmonic modes of different order. The waveguide refractive index profiles in two orthogonal planes are shown by the solid and dashed lines. (b) Microscope photograph of the cross section of a Ba/Rb-exchanged channel waveguide in a KTP crystal [10].

\section{EXPERIMENTAL SETUP}

For this work, an experimental setup, as shown in Figure 2, was used. The laser pump source consisted of a QD gain chip of $4 \mathrm{~mm}$ total length with 1-mm absorber, similar to the described in [9]. The active region of the gain chip contained 10 non-identical InAs QD layers, incorporated into $\mathrm{Al}_{0.35} \mathrm{Ga}_{0.65} \mathrm{As}$ cladding layers and grown on a GaAs substrate by molecular beam epitaxy. The gain chip ridge waveguide was angled at $7^{\circ}$ relative to the normal of the AR-coated back facet (both facets had conventional AR coatings, resulting in total estimated reflectivities of $10^{-2}$ for the front facet and less than $10^{-5}$ for the angled facet). The gain chip was set-up in a quasi-Littrow configuration [6], whereby a diffraction grating with 1200 grooves $/ \mathrm{mm}$ was used to provide the first order diffraction beam back to the gain chip, and thus enabling to achieve a broad wavelength tunability.

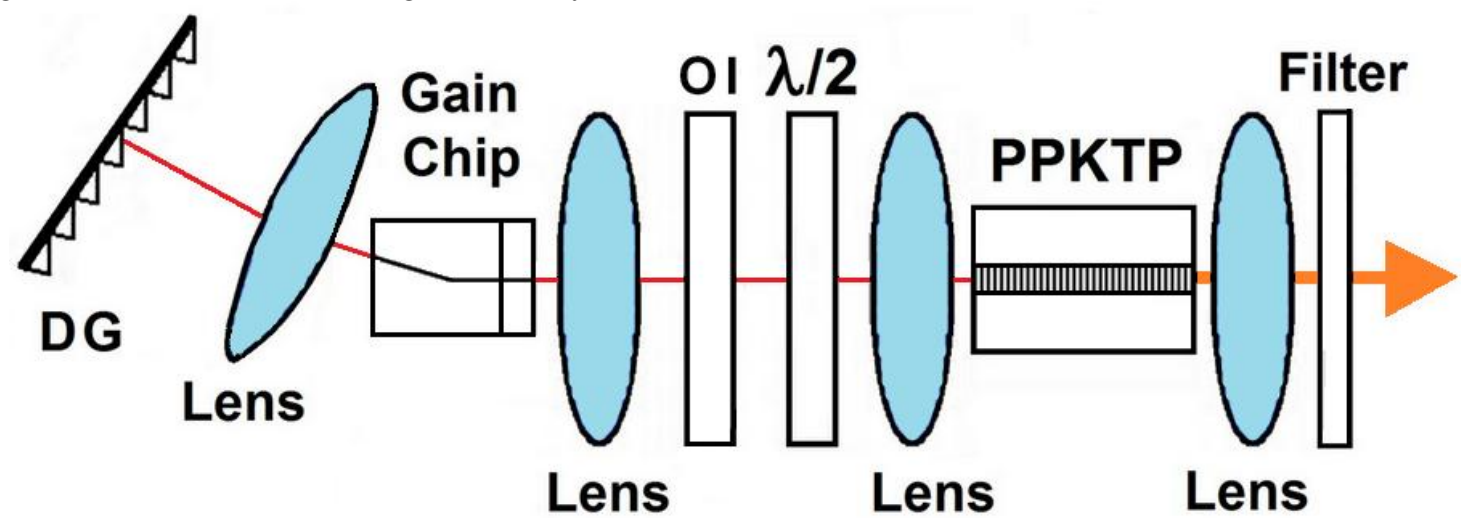

Figure 2. Simplified schematics of the experimental setup consisting of diffraction grating (DG), 40x AR-coated aspheric lenses, QD gain chip, optical isolator $(\mathrm{OI})$, half-wave plate $(\lambda / 2)$, PPKTP waveguide and filter. 
Coarse wavelength tuning between $1193 \mathrm{~nm}$ and $1284 \mathrm{~nm}$ was possible for pump current of $900 \mathrm{~mA}$ and reverse bias of $3 \mathrm{~V}$. Pulsed operation was observed at any wavelength. The laser output was coupled into the uncoated PPKTP waveguide (Figure 1 (b)) with the poling period of $\sim 13.82 \mu \mathrm{m}$, which was $13 \mathrm{~mm}$ in length and had a cross-sectional area of $\sim 4 \times 4 \mu \mathrm{m}^{2}$. The waveguide was fabricated by ion-exchange technique that provided a refractive index step of $\sim 0.01$ [7]. Both the pump laser and the PPKTP crystal were operating at room temperature. The frequency-doubled output light was then collected by a power meter after a suitable filter at the fundamental wavelength.

\section{EXPERIMENTAL RESULTS}

Tunable picosecond second-harmonic generation in the spectral region between $600 \mathrm{~nm}$ and $627 \mathrm{~nm}$ was achieved for the laser output tuned across the $1200 \mathrm{~nm}-1254 \mathrm{~nm}$ wavelength range under an applied constant current of $900 \mathrm{~mA}$ and reverse bias of $3 \mathrm{~V}$. The optical spectra of efficient second harmonic generation at $600 \mathrm{~nm}, 613 \mathrm{~nm}$ and $627 \mathrm{~nm}$ are shown in Figure 3. The autocorrelation signals and the corresponding RF spectra for frequency-doubled light at these wavelengths are shown in Figure 4 (a-f). A measured Gaussian fitting pulse shape resulted in deconvolved pulse duration varied between $14.7 \mathrm{ps}$ and $29.3 \mathrm{ps}$ across the tuning range under a constant bias condition.

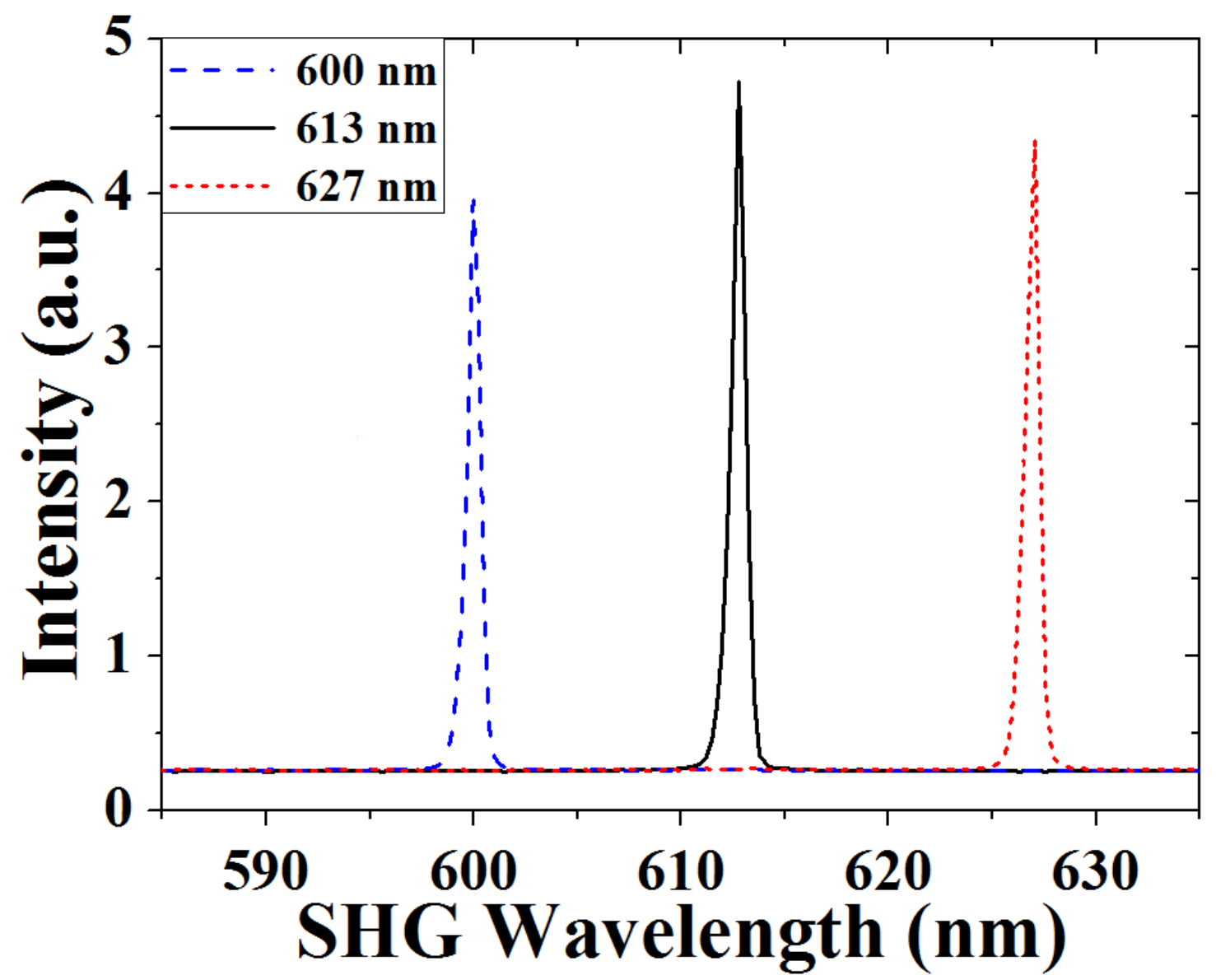

Figure 3. Optical spectra of the second harmonic generation at the wavelengths: $600 \mathrm{~nm}, 613 \mathrm{~nm}$ and $627 \mathrm{~nm}$. 

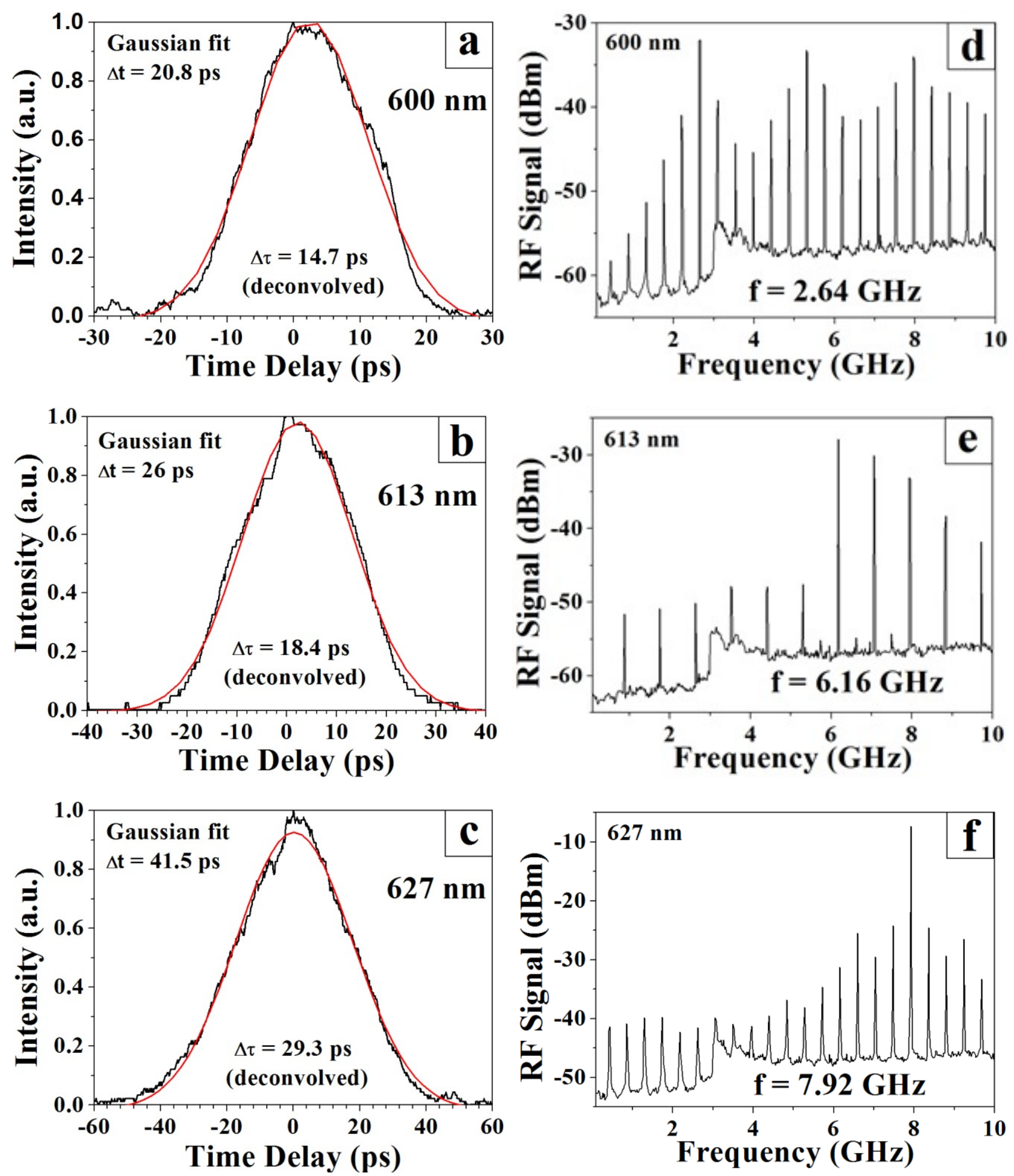

Figure 4. Autocorrelation signals and corresponding RF spectra at $600 \mathrm{~nm}, 613 \mathrm{~nm}$ and $627 \mathrm{~nm}$. 
The maximum SHG output peak power of $3.25 \mathrm{~mW}$ at $613 \mathrm{~nm}$ was achieved for $71.43 \mathrm{~mW}$ of launched pump peak power at $1226 \mathrm{~nm}$, resulting in conversion efficiency of $4.55 \%$ (Figure 5). SHG at $600 \mathrm{~nm}$ and $627 \mathrm{~nm}$, which corresponded to phase-matching between fundamental and SHG modes of different order, was demonstrated with output peak power of $0.95 \mathrm{~mW}$ and $0.66 \mathrm{~mW}$ and with conversion efficiency of $1.5 \%$ and $0.92 \%$, respectively. A photograph of the second harmonic generation in the PPKTP waveguide pumped by the tunable QD-ECMML is shown in Figure 6.

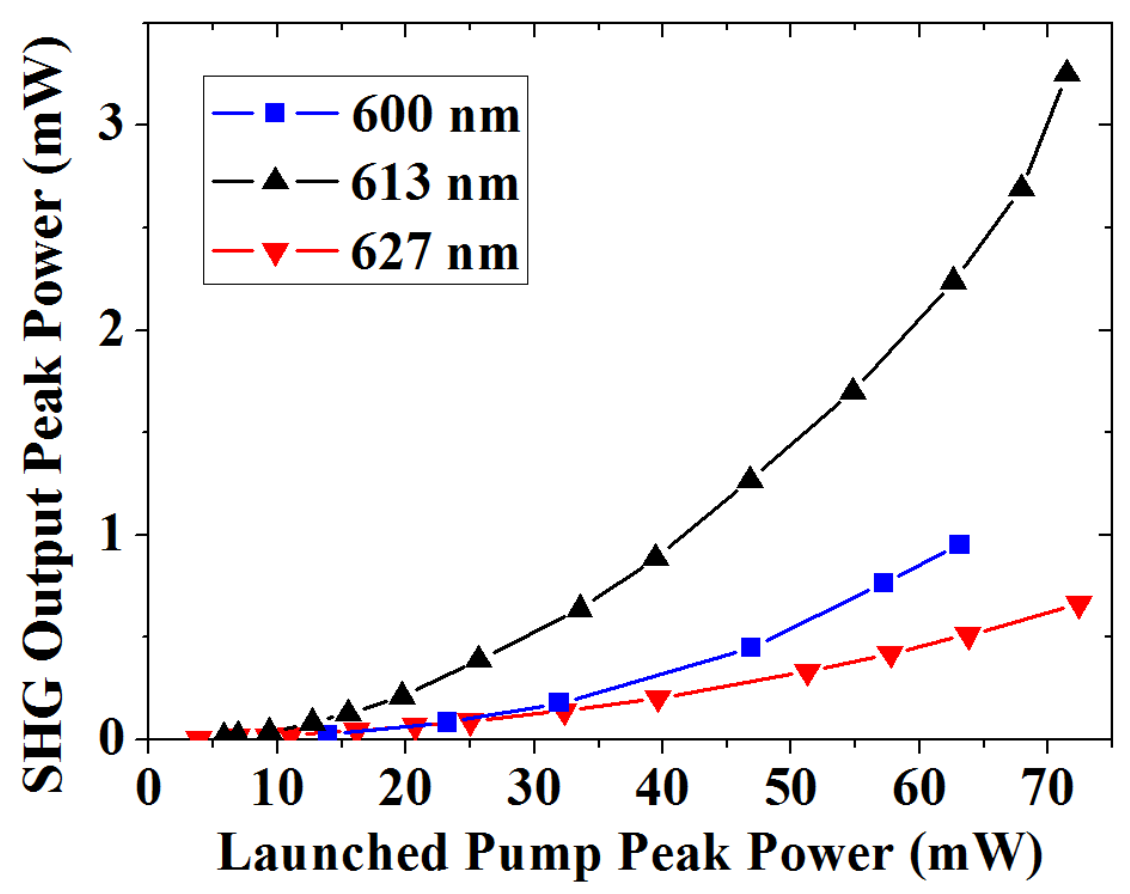

Figure 5. Frequency-doubled output peak power versus launched pump power for $600 \mathrm{~nm}, 613 \mathrm{~nm}$ and $627 \mathrm{~nm}$.

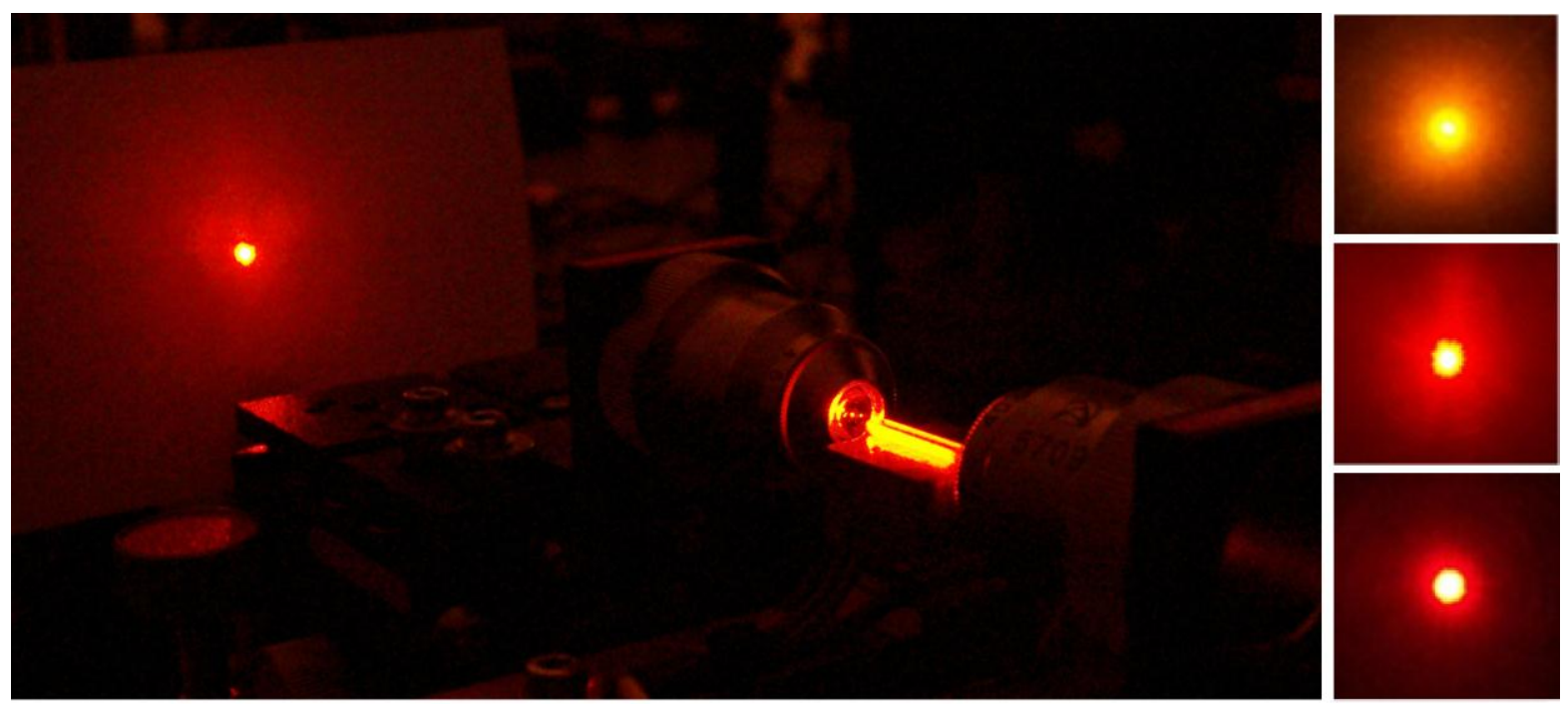

Figure 6. Efficient second harmonic generation at $613 \mathrm{~nm}$ from a PPKTP waveguide end-pumped by a quantum-dot tunable mode-locked laser. 


\section{CONCLUSSION}

Tunable all-room-temperature picosecond laser source in the visible spectral region (between $600 \mathrm{~nm}$ and $627 \mathrm{~nm}$ ) based on a single widely tunable QD diode laser and a single PPKTP waveguide was demonstrated by utilization of a significant difference in the effective refractive indices of the high-order and low-order modes in multimode waveguide. A frequency-doubled peak power of up to $3.25 \mathrm{~mW}$ at the wavelength of $613 \mathrm{~nm}$ was achieved. This represents a practical broadly tunable visible laser source operating in the picosecond regime which is of considerable interest for a wide range of applications. Further work on the improvement of SHG conversion efficiency and further extension of tunability is currently under way.

\section{ACKNOWLEDGEMENT}

This work was partly supported by the EU FP7 programme through the FAST-DOT project (contract no. 224338). G. S. Sokolovskii acknowledges financial support from a Marie Curie Fellowship (Sendbeam project).

\section{REFERENCES}

[1] Palumbo,G., Pratesi, R., "Lasers and Current Optical Techniques in Biology," from Comprehensive Series in Photochemistry and Photobiology, Vol. 4, Springer (2004).

[2] Choudhary, S., Nouri, K., Elsaie, L., "Photodynamic therapy in dermatology: a review," Lasers Med. Sci., 24, 971-980 (2009).

[3] Baratto, L., Calza, L., Capra, R., Gallamini, M., Giardino, L., Giuliani, A., Lorenzini, L., Traverso, S., "Ultralow-level laser therapy," Lasers Med. Sci., 26, 103-112 (2011).

[4] Jansen, M., Carey, G. P., Carico, R., Dato, R., Earman, A. M., Finander, M.J., Giaretta, G., Hallstein, S., Hofler, H., Kocot, C.P., Lim, S., Krueger, J., Mooradian, A., Niven, G., Okuno, Y., Patterson, F.G., Tandon, A., Umbrasas, A., "Visible Laser Sources for Projection Displays," Proc. of SPIE, vol. 6489, 648908 (2007).

[5] Fedorova, K. A., Cataluna, M. A., Battle, P. R., Kaleva, C. M., Krestnikov, I. L., Livshits, D. A., Rafailov, E.U., "Orange light generation from a PPKTP waveguide end pumped by a cw quantum-dot tunable laser diode," Appl. Phys. B, 103(1), 41-43 (2011).

[6] Fedorova, K. A., Cataluna, M. A., Krestnikov, I., Livshits, D. and Rafailov, E. U., "Broadly-Tunable HighPower InAs/GaAs Quantum-Dot External-Cavity Diode Lasers," Opt. Express, 18 (18), 19438-19443 (2010).

[7] Fedorova, K. A., Sokolovskii, G. S., Battle, P. R., Livshits, D. A., Rafailov, E. U., "Green-to-red tunable SHG of a quantum-dot laser in a PPKTP waveguide," Laser Physics Letters, 9, 790-795 (2012).

[8] Rafailov, E. U., Cataluna, M. A., Sibbett, W., "Mode-locked quantum-dot lasers," Nature Photonics, 1, 395 (2007).

[9] Nikitichev, D. I., Fedorova, K. A., Ding, Y., Alhazime, A., Able, A., Kaenders, W., Krestnikov, I., Livshits, D., Rafailov, E. U., "Broad wavelength tenability from external cavity quantum-dot mode-locked laser," Applied Physics Letters, 101, 121107 (2012).

[10] http://www.advr-inc.com 